\title{
Electro paramagnetic resonance and magnetization measurements of metal-substituted hydroxyapatites used in hyperthermia applications
}

\author{
H. Kamal \\ Physics Department, Faculty of Science, Mansoura University, Mansoura 35516, Egypt \\ hkamal2000@gmail.com
}

\begin{abstract}
Pure and metal doped hydroxyapatite samples nano-particles were prepared by the wet chemical method. Copper and cobalt is used in doping hydroxyapatite. Sample was prepared without change in the stoichiometric ratio of $\mathrm{Ca} / \mathrm{P}$ and $\mathrm{Ca}+\mathrm{M} / \mathrm{P}$ inside the structure of $\mathrm{HA}(\mathrm{M}$;metal). Sample was characterized by electron paramagnetic resonance, magnetization, transmission electron microscope and electron diffraction. Samples posses the highest value of magnetic susceptibility was chosen for more study to test their ability for application in the field of hyperthermia treatment of bone tumors. Magnetization curves were obtained for samples to study their behavior under the effect of magnetic field. The sample doped with copper and cobalt exhibited hysteresis loops which are characteristic for the magnetic materials. The samples were classified to be ferromagnetic material. Sample prepared by mixing $\mathrm{Cu}$ and co had the highest values of saturation magnetization (MS), area (A) enclosed within the hysteresis loop and magnetic anisotropy which represent an indicator of the energy generated in the material under the effect of magnetic field, and hence the amount of heat produced by the sample. TEM and EDP techniques were used to study the internal structure of these samples. The micrographs and the diffraction patterns showed and confirmed the presence of crystal structures within the samples. The particle size was calculated from the micrographs and found to be in the range of nanometer for all the selected samples.
\end{abstract}

\section{KEY WORDS: - hydroxyapatite - metal doped- EPR - magnetization and hyperthermia INTRODUCTION}

Hydroxyapatite $(\mathrm{HA})\left(\mathrm{Ca}_{10}\left(\mathrm{PO}_{4}\right)_{6}(\mathrm{OH})_{2}\right)$ ceramic implants have attracted much attention as an alternative substance for autogenous free bone grafting [1-3]. It is the most prominent bioactive ceramics and is widely used and investigated [4]. Applications include coatings of orthopedic and dental implants, alveolar ridge augmentation, maxillofacial surgery, otolaryngology, and scaffolds for bone growth and as powders in total hip and knee surgery [5]. Synthetic HA is classified as polycrystalline ceramics since its material structure is derived from individual crystals that have been fused together by a high temperature sintering [6].

Incorporation of Magnetic Phases into Hydroxyapatite: Because of the importance of having non - toxic effect for the purposes bone hyperthermia, recent researches focused on the development of biocompatible and bioresorbable superparamagnetic phase by doping hydroxyapatite with magnetic elements, such as iron. This new apatite will have magnetic properties and it will also represent a new type of scaffold for bone tissue regeneration [7-8].

Magnetic apatite represents a suitable biocompatible and non-toxic alternative for magnetite in case of magnetic based hyperthermia treatments [9]. Hyperthermia includes raising the temperature at the tumour site for limited periods of time, which is considered an effective treatment for bone cancer. Tumour cells can be destroyed by applying an external magnetic field on MNPs deliberately placed near the tumour tissue [10]. Although iron is a very important element in the human body, its concentration within bone is low. On the other hand, HA is well known for its biocompatibility and bioactivity, and in fact most of the existing bone graft scaffolds consist of calcium phosphate-based materials [11]. From this point of view, hydroxyapatite doped with iron represents a very promising material for application either as bioactive material for bone tissue regeneration or as nontoxic magnetic nanomaterial [12].

Authors described the preparation of hydroxyapatite using chemical precipitation method [13]. The precursor materials were calcium nitrate and diammonium hydrogen phosphate. Since then, many methods were developed for preparation of hydroxyapatite. After that, many researches were carried out to improve the quality of the apatite material produced via chemical precipitation. Liu et. al. studied the effect of changing the temperature and the $\mathrm{pH}$ of the solution during preparation [12]. Changing these parameters was found to change the morphology of the hydroxyapatite crystal. Tas reported a synthesis method that mimics the formation of hydroxyapatite in the human body [14]. This method is based on dissolving the starting materials in simulated body fluid, instead of water, to provide conditions very similar to that of the body environment. Paramanik et. al. used solid state sintering method to prepare hydroxyapatite and also studied the structural changes in a wide range of temperatures [13]. Another method of preparation is the hydrolysis method, in which calcium phosphate phases, such as octacalcium phosphate, are converted into hydroxyapatite [15]. In fact, this method does not have much significance as the rate of conversion of octacalcium phosphate is very slow. R.Z. LeGeros conducted many research on the biological activity of hydroxyapatite and calcium phosphate biomaterials [16]. She discussed in details the osteoinductive properties of different calcium phosphate ceramics, and also the behavior of these materials in cellular environment. She also studied the effect of substitutions in the apatite crystal and incorporations of different ions on the structure and properties of hydroxyapatite. The incorporation of fluoride and carbonate into apatite crystal enhances the solubility of hydroxyapatite and hence enhances its bioactivity.

The incorporation of some cations into the apatite crystal was found to improve the biological activity. The addition of magnesium and zinc enhanced the properties of newly formed bones in vivo. Also silicon can be incorporated into apatite crystal, as it is considered an essential trace element in bone growth. The addition of silicon to hydroxyapatite with relatively low concentrations was found to have a positive effect on the osteoblasts activity in vitro [17]. 
Another element that could be incorporated into the apatite crystal structure is strontium. Strontium has a useful effect in the treatment of osteoporosis. O'Donnell et. al. studied the structural changes in the apatite crystal due to addition of strontium [18]. The biological activity of hydroxyapatite was enhanced by addition of strontium. Apatite doped with low concentrations of strontium exhibited an increase in the activity and the number of osteoblasts in vitro. Manganese can also be incorporated into hydroxyapatite as it influences bone remodeling and its deficiency may cause abnormalities in bone growth. Manganese doped hydroxyapatite was shown to induce the formation and growth when used as a coating on the surface of titanium implants [19]. High contents of manganese added to hydroxyapatite resulted in small, non stoicheometric apatite crystals, increasing the biocompatibility and resorbability. For the purpose of hyperthermia treatments of bone cancer, many ions can be incorporated into hydroxyapatite to provide good magnetic properties without affecting its biocompatibility [20].

The most common element used for this application is iron. Iron oxide can be incorporated with hydroxyapatite by the co -precipitation method, producing a composite material of hydroxyapatite and iron oxide. Another approach was reported depending on coating magnetite nanoparticles with hydroxyapatite [21]. It was found that the samples coated with hydroxyapatite increased the osteoblasts activity. Co-precipitation method was used to synthesize iron and manganese substituted hydroxyapatite, resulted in nanoparticles having core and shell structure Iron doped hydroxyapatite prepared by co-precipitation method was found excellent hyperthermia effect in vivo [21]. The samples were injected into mice, hyperthermia was achieved by using alternating magnetic field and there was an obvious decrease in the tumor size in only 15 days [22].

The aim of this study is to explore the magnetic properties of some metal doped hydroxyapatite samples for the use in tumors treatment. The new types of these materials appear best suited for potential use as hyperthermia treatments. Also, the present investigation was attempted to develop new compositions that would be as safe and effective. These apatites represent new types of scaffold for bone tissue regeneration.

\section{MATERIALS AND METHODS}

Compositions of the Prepared Samples: The chemical formula of hydroxyapatite is $\mathrm{Ca}_{10}\left(\mathrm{PO}_{4}\right)_{6}(\mathrm{OH})_{2}$ with Stoichiometric $\mathrm{Ca} / \mathrm{P}$ ratio $=10 / 6(\sim 1.67)$. The samples were prepared with the formula $\mathrm{Ca}_{10-\mathrm{x}} \mathrm{M}_{\mathrm{x}}\left(\mathrm{PO}_{4}\right)_{6}(\mathrm{OH})_{2}$ where $\mathrm{M}$ is the metal used. In this case the ratio is $(\mathrm{Ca}+\mathrm{M}) / \mathrm{P} \sim 1.67$.

The materials used in the preparation of the samples are calcium nitrate tetrahydrate $\left(\mathrm{Ca}\left(\mathrm{NO}_{3}\right)_{2} .4 \mathrm{H} 2 \mathrm{O}\right)$, diammonium hydrogen orthophosphate $\left(\left(\mathrm{NH}_{4}\right)_{2} \mathrm{HPO}_{4}\right)$. Cupper chloride dihydrate $\left(\mathrm{CuCl}_{2} \cdot 2 \mathrm{H}_{2} \mathrm{O}\right)$ was used as the source of cupper, while cobalt chloride hexahydrate $\left(\mathrm{CoCl}_{2} \cdot 6 \mathrm{H}_{2} \mathrm{O}\right)$ was used as the source of cobalt. Ammonia solution $(33 \%)$ was used for controlling the $\mathrm{pH}$ of reaction at about 9 . Reagents were dissolved in distilled water. The calcium nitrate solution was heated to $80^{\circ} \mathrm{C}$ on a heater - stirrer. Diammonium hydrogen orthophosphate solution was added drop wise with continuous stirring for one hour. The solutions were then kept in a water bath at $37^{\circ} \mathrm{C}$ for 24 hours. In this stage of preparation, the samples were observed to contain a precipitate layer and a clear supernatant layer. The precipitates were collected, filtered and washed many times with distilled water to remove excess ammonia. The samples were then dried at $100{ }^{\circ} \mathrm{C}$ to remove excess water. Heat Treatment: The blank sample (HAp) was heat-treated in a furnace at temperature $700{ }^{\circ} \mathrm{C}$. The samples were into the furnace and held at the temperature of heat treatment for one hour then they were cooled normally to room temperature. Then all the samples containing copper and cobalt were heat-treated in the same way at temperature $700{ }^{\circ} \mathrm{C}$ for one hour.

Electron Paramagnetic Resonance (EPR) Spectrometry: The presence of unpaired electrons in the prepared samples is detected via EPR spectroscopy. Resonance signals were recorded at room temperature by X-band EMX spectrometer (Bruker, Germany). The samples were filled into a fine silica tube. The tube is inserted into a standard ER 4102 rectangular cavity. The instrument was operated at frequency of $100 \mathrm{kHz}$ and the signals were recorded.

Magnetic Susceptibility Calculations: Magnetic susceptibility was measured using Sherwood magnetic susceptibility balance. A known weight of the sample is placed in a clean silica capillary tube. The length and the weight of the sample were determined. Then the sample is placed in the instrument and the reading is recorded. The reading represents the "calibration constant of magnetic balance". Magnetic Susceptibility was calculated from the equation [23]:

$$
\chi_{V}=\frac{\left(R-R_{0}\right) \times C x L}{\left(W-W_{o}\right) \times 10^{9}}
$$

Where $\chi_{v}$ is volume susceptibility, $\left(\mathrm{R}^{\circ}\right)$ is the record of the instrument without the sample which equals $-38,(\mathrm{R})$ is the record with the sample, $(C)$ is the balance constant which equals $0.0259,(L)$ is the height of the sample in the tube, $\left(\mathrm{W}^{\circ}\right)$ is the weight of the empty tube and (W) is the weight of the tube with the sample.

Magnetization Measurements (VSM): The magnetic properties of chosen samples were studied using a 7400 Lake Shore Vibrating Sample Magnetometer (VSM). The sample was filled into a tube and then placed between two electromagnet poles. Magnetic field is applied and the sample is moved up and down in vicinity of detection coils, inducing an electric current in the coils. The induced electric current is measured to find the magnetic moment of the sample. The measurement was carried at room temperature. The magnetic field range was 0-2 tesla.

Transmission Electron Microscope (TEM) and Electron Diffraction Pattern (EDP): Transmission electron micrographs and their electron diffraction patterns were obtained using a JEOL JEM-2100 Electron Microscope. Powdered 
samples were suspended in distilled water using MCS Digital Ultrasonic Heater. Then the sampled were loaded on a grid and let to dry. The sample is then inserted into the instrument and examined. The electron beam interacts with the sample and the transmitted part is processed to form images of the sample.

\section{RESULTS AND DISSCUSION}

It is well known that the total magnetic field of each ion is established from the spin-spin interaction of neighboring paramagnetic ions. The spin-spin interaction caused by small magnetic fields and this play an important role to alter the total magnetic field of each ion. So, the energy levels of the unpaired electrons to be shifted which led to a variation of energies resulting in a broadened EPR signal.

Figures (1), (2), (3) and (4) show the EPR spectra of undoped HA, Cu- doped HA samples, Co- doped HA samples and $\mathrm{Cu}$, Co doped HA respectively. Table (1) indicates the g-factor values corresponding to each sample.

Table (1): The values of $g$-factor of the prepared samples

\begin{tabular}{|c|c|c|c|c|c|}
\hline $\begin{array}{c}\text { Prepared } \\
\text { sample }\end{array}$ & $\begin{array}{c}\text { g- } \\
\text { factor }\end{array}$ & $\begin{array}{c}\text { Prepared } \\
\text { sample }\end{array}$ & $\begin{array}{c}\text { g- } \\
\text { factor }\end{array}$ & $\begin{array}{c}\text { Prepared } \\
\text { sample }\end{array}$ & $\begin{array}{c}\text { g- } \\
\text { factor }\end{array}$ \\
\hline $\mathrm{HA}$ & 1.61 & ----- & ---- & ---- & ---- \\
\hline $\mathrm{Cu}-5$ & 1.99 & $\mathrm{Co}-5$ & 1.85 & $\mathrm{Co} 1.25-\mathrm{Cu} 2.5$ & 1.77 \\
\hline $\mathrm{Cu}-10$ & 1.90 & $\mathrm{Co}-10$ & 1.97 & $\mathrm{Co} 2.5-\mathrm{Cu} 5$ & 1.86 \\
\hline $\mathrm{Cu}-15$ & 1.93 & $\mathrm{Co}-15$ & 1.89 & $\mathrm{Co5}-\mathrm{Cu} 10$ & 1.98 \\
\hline $\mathrm{Cu}-20$ & 1.68 & $\mathrm{Co}-20$ & 1.80 & ----- & ---- \\
\hline
\end{tabular}

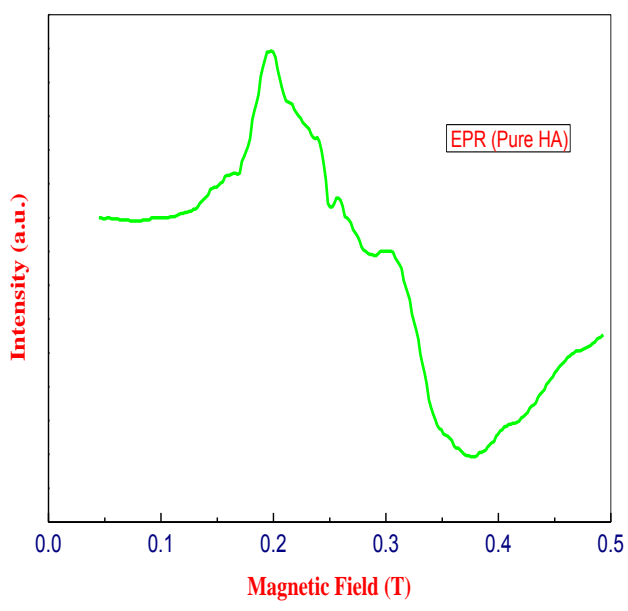

Figure 1:- EPR spectrum for pure hydroxyapatite

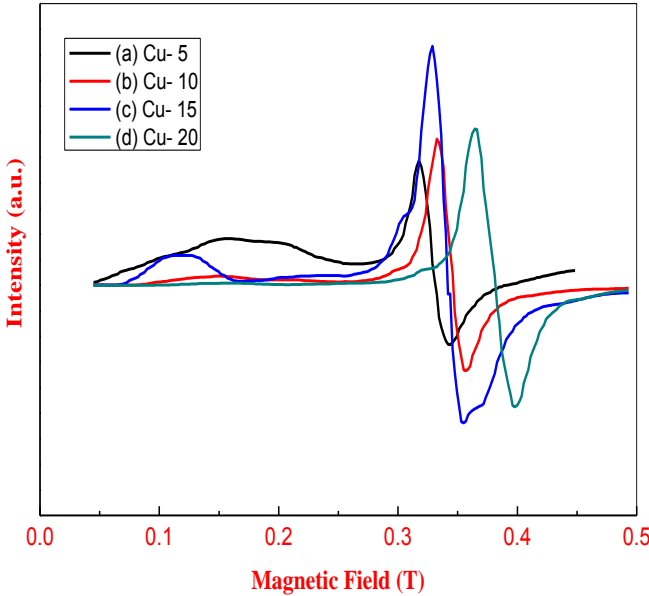

Figure 2:- EPR spectrum for $\mathrm{Cu}$ - doped hydroxyapatite samples 


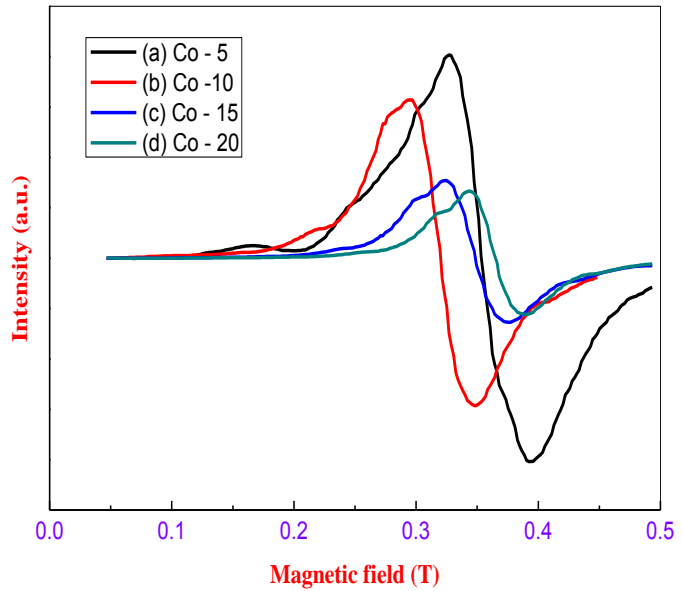

Figure 3:- EPR spectrum for Co- doped hydroxyapatite samples

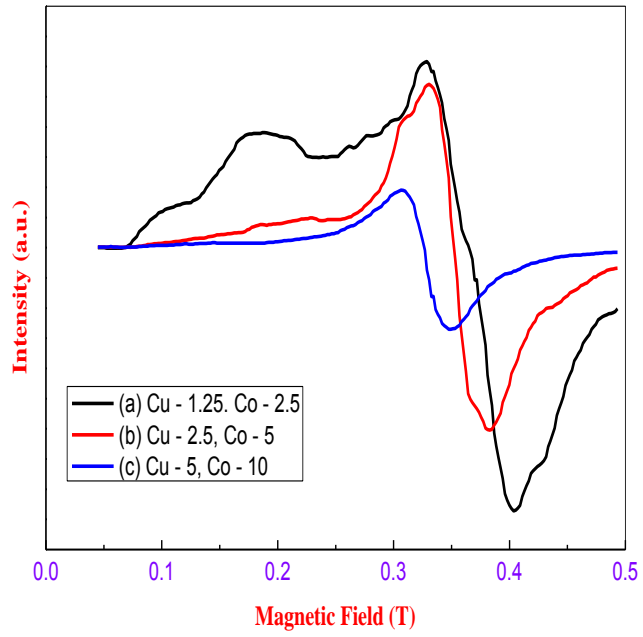

Figure 4:- EPR spectrum for $\mathrm{Cu}$ and Co doped hydroxyapatite samples

In $\mathrm{Cu}-\mathrm{HA}$ sample, the broad feature in the $\mathrm{g}$ of 2.0 regions is attributed to separation of copper oxide phases with different content in $\mathrm{Cu}$ cations, and their separation results presence of surface oxyhydroxides and Cu-O-Cu clusters [24]. The presence of high concentration of cupper caused electron spin-spin interactions between neighboring cupper nuclei which yielded the broad $\mathrm{g}=2.0$ signal. The poor crystallinity with sample $\mathrm{Fe}-\mathrm{HA}$ that results from the $\mathrm{Cu}$-phosphates and $\mathrm{Cu}$ oxyhydroxides would be expected to have a high concentration of neighboring cupper ions [25]. The number and position of EPR transitions for isolated cupper ions in a site of well-defined symmetry observable in a powder spectrum depends sensitively on the local ligand-field symmetry of the sites and possible magnetic interactions between them. In the presence of a rhombic distortion of the ligand-field the $g$ values of the three doublets of the $S=5 / 2$ system change as a zero-field splitting parameters depending of its value it becomes possible to observe ESR signal arising from the $\pm 3 / 2$ and $\pm 5 / 2$ doublet of the excited states. Cobalt may be the cause of the low field X-band hyperfine patterns. Cobalt would have originated from cobalt oxidation during Co-HA synthesis. However, the pink color of the Co-HA materials indicated that cobalt was the dominant oxidation state in the g-value 2 [26].

The hyperfine lines of the Co-HA were barely detectable. Minor spectral differences were observed between the Co-HA and $\mathrm{Cu}-\mathrm{HA}$ showed a marked decrease in peak intensity relative to standard HA. The large decrease of the Co-HA g 2.01 peak indicated that nonstructural Co phase(s) such as poorly crystalline Co-oxyhydroxide and Co-phosphate phase(s) associated with the HA crystallites. Poorly crystalline Co phase(s) also explain the broad g-value 2.01 peak in Co-HA that was superimposed on the hyperfine pattern because cobalt would be closely associated with one another leading to spinspin interactions [25]. The high intensity of the cobalt peaks Co-HA dominated the spectrum and masked any contamination peak [100]. The entire Cu-HA spectrum was superimposed on the broad signal that suggested the presence of sites with strong spin-spin interactions caused by poorly crystalline Cu-oxyhydroxide or Cu-phosphate phase(s)[25].

Magnetic Susceptibility: Magnetic susceptibility is defined as the ability of the material to be magnetized in the presence of magnetic field [27]. Figures (6), (7) and (8) represent the relation between the concentration of the doping agent and the magnetic susceptibility of the sample. The value of magnetic susceptibility of the undoped hydroxyapatite sample is very close to zero. This is a predictable result, as hydroxyapatite is classified as a diamagnetic material. It can be noticed that the value of magnetic susceptibility increases linearly with increasing the concentration of the doping agent. This increase is attributed to the incorporation of the magnetic elements into the apatite crystal structure and also the formation of magnetic phases. Between the orders of $10^{-6}$ and $10^{-7}$, indicating a paramagnetic behavior. The samples with high values of magnetic susceptibility, especially with values in the order of $10^{-6}$ are considered as good candidates for the hyperthermia application [28].

Magnetization Measurements: The magnetization curves describe the behavior of the material under the effect of applied magnetic field. The magnetic properties of any material can be deduced from the curve of magnetization vs. field, which is also known as "( $\mathrm{M}-\mathrm{H})$ hysteresis loop". Figure (5) illustrates a schematic hysteresis loop. 


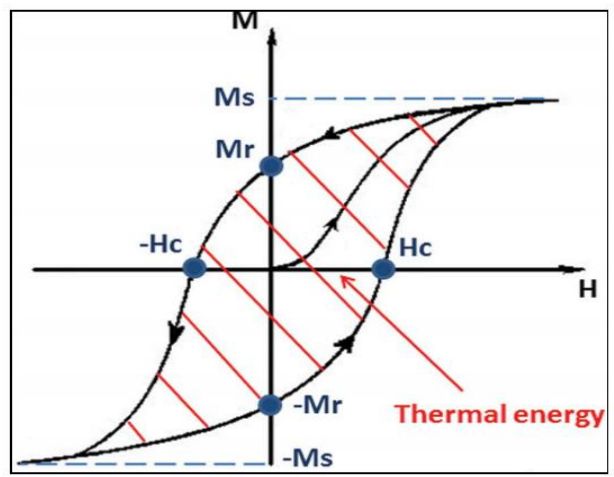

Figure 5:- Hysteresis loop of a ferromagnetic sample. The area of the hysteresis loop represents the energy dissipated during a magnetization cycle.

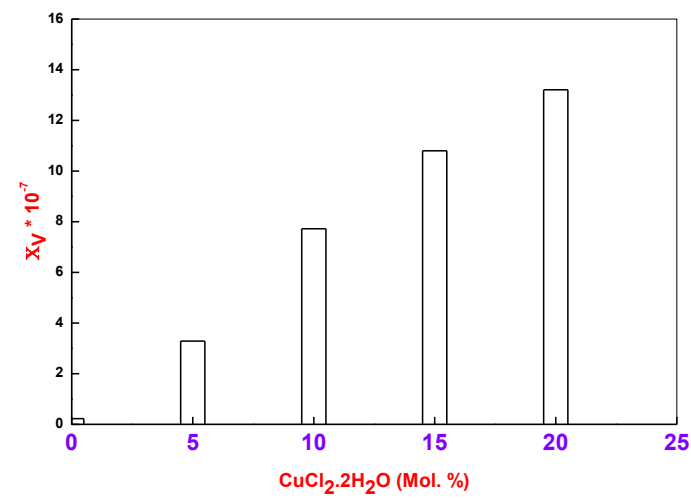

Figure 6:- Volume Susceptibility of Cu doped hydroxyapatite samples.

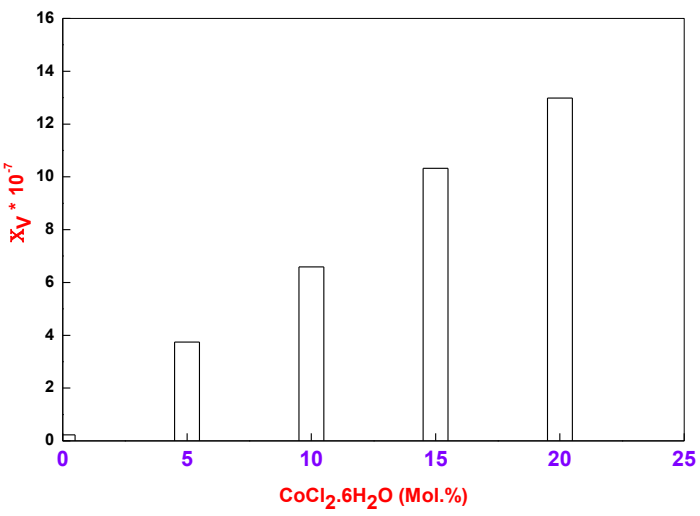

Figure 7:- Volume Susceptibility of Co doped hydroxyapatite samples.

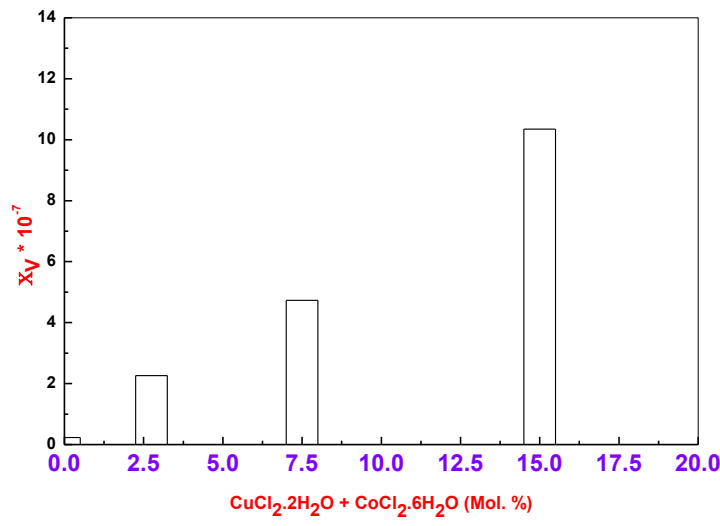

Figure 8:- Volume Susceptibility of $\mathrm{Cu}$ and Co doped hydroxyapatite samples. 
There are 3 characteristic values for magnetic materials that can be defined from the $M$ versus $H$ hysteresis loop [29] These values are: Saturation magnetization (MS), which represents the maximum value of magnetization that occurs when the magnetic moments inside the material are aligned in a parallel direction with the external magnetic field [29]. Remanent magnetiation (MR), represents the remaining magnetization in the sample when the external magnetic field is removed. Coercivity (or Coercive Field) $(\mathrm{HC})$, which is defined as the magnetic field that should be applied to restore the value of magnetization back to zero [29]. There are also other parameters that are very important in the application of hyperthermia, such as the area enclosed within the curve $(A)$, and the anisotropy energy density (K). These parameters can be calculated from the magnetization curve [30].

The anisotropy energy density, $\mathrm{K}$, is defined as "the energy required changing the magnetization of the material with respect to its physical structure" [31]. In other words, if a material exhibits a preferred direction of the magnetic moment with respect to other physical parameters, such as crystal orientation, it is said to exhibit anisotropy. The energy required to change this orientation is called the anisotropy energy. It is dependent on the anisotropy field $\left(\mathrm{H}_{\mathrm{K}}\right)$ or "the magnetic field required to completely changing the magnetization direction" [32]. Its value can be calculated from the equation:

$$
\mu_{0} H_{K}=\frac{2 K}{M_{S}}
$$

Where $\mu_{\circ}$ is the permeability of free space, which equals $4 \pi$, and MS is the saturation magnetization. Also the area enclosed within the hysteresis loop is an important factor, as it represents the amount of heat generated from hysteresis loss [29]. The area can be calculated by integration of the region within the magnetization curve. This integration is a mathematical representation of the work done to magnetize the material. Since there is no change in the internal energy, all the work is converted into thermal energy [29]. The samples with the highest values of magnetic susceptibility were chosen to study their hysteresis behavior.

Figures (9), (10) and (11) represent magnetization behavior of samples doped with cupper, cobalt and both cupper and cobalt respectively. The magnetization behavior of the undoped hydroxyapatite sample was also recorded for comparison. As noticed, all the samples gave a typical hysteresis loop with a variation in the characteristic parameters of the curve. It is obvious that the magnetic saturation value of the sample (Co-15) is slightly higher than that of sample (Co-20). It is also obvious that sample (Co-5, Cu-10) has the highest saturation magnetization, with value of $3.30 \mathrm{~A} . \mathrm{m}^{2} / \mathrm{Kg}$. The values of $\mathrm{MR}, \mathrm{MS}$ and $\mathrm{HC}$ are listed in table (2).

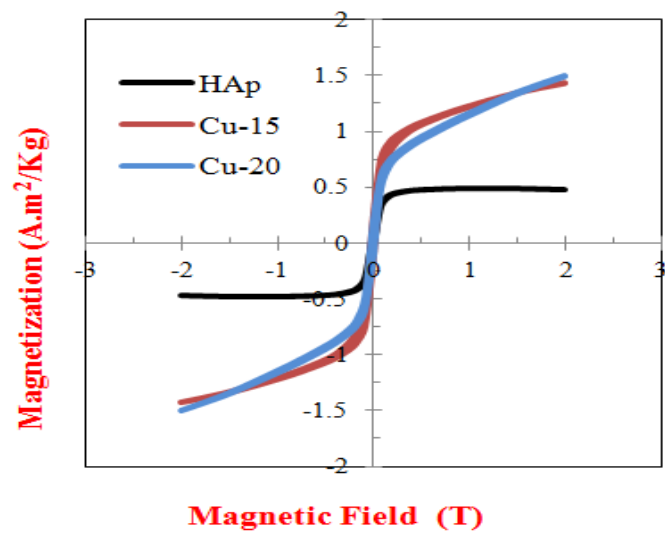

Figure 9:- Magnetization curve of Cu doped hydroxyapatite and pure hydroxyapatite

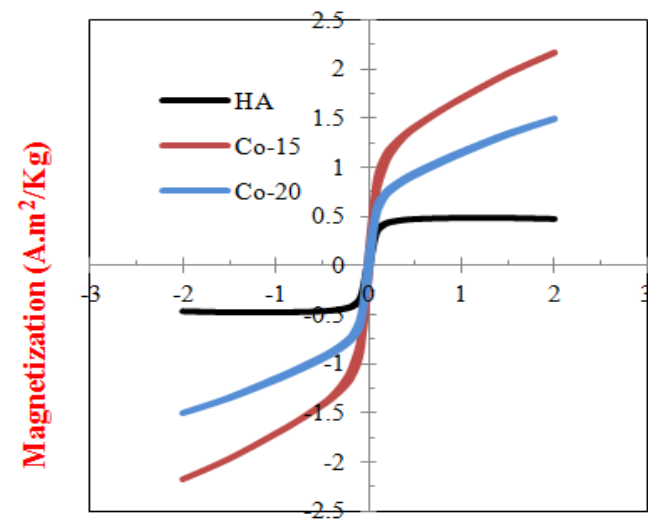

Magnetic Field (T)

Figure 10:- Magnetization curve of Co doped hydroxyapatite and pure hydroxyapatite 


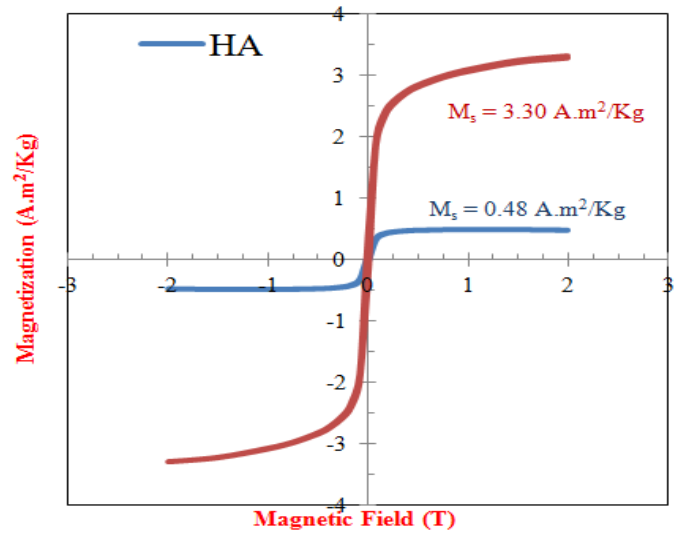

Figure 11:- Magnetization curve of $\mathrm{Cu}$ and $\mathrm{Co}$ doped hydroxyapatite and pure hydroxyapatite

Table (2):-Values of magnetic saturation (Ms), Remanence magnetization and Coerecivity calculated from previous magnetization curves.

\begin{tabular}{|c|c|c|c|}
\hline Sample & $\begin{array}{c}\mathrm{M}_{\mathrm{S}} \\
\left(\mathrm{A} \cdot \mathrm{m}^{2} / \mathrm{Kg}\right)\end{array}$ & $\begin{array}{c}\mathrm{M}_{\mathrm{R}} \\
\left(\mathrm{A} \cdot \mathrm{m}^{2} / \mathrm{Kg}\right)\end{array}$ & $\begin{array}{c}\mathrm{H}_{\mathrm{C}} \\
(\mathrm{T})\end{array}$ \\
\hline $\mathrm{HA}$ & $0.48 \pm 0.02$ & $0.06 \pm 0.01$ & $0.013 \pm 0.001$ \\
\hline $\mathrm{Cu}-15$ & $1.43 \pm 0.04$ & $0.18 \pm 0.01$ & $0.0194 \pm 0.001$ \\
\hline $\mathrm{Cu}-20$ & $1.40 \pm 0.04$ & $0.15 \pm 0.01$ & $0.0198 \pm 0.001$ \\
\hline $\mathrm{Co}-15$ & $2.18 \pm 0.06$ & $0.20 \pm 0.01$ & $0.0186 \pm 0.001$ \\
\hline $\mathrm{Co}-20$ & $1.67 \pm 0.05$ & $0.15 \pm 0.01$ & $0.0180 \pm 0.001$ \\
\hline $\mathrm{Co}-5, \mathrm{Cu}-10$ & $3.31 \pm 0.08$ & $0.38 \pm 0.02$ & $0.0139 \pm 0.001$ \\
\hline
\end{tabular}

In case of sample (Co-5, Cu-10), the high value of saturation magnetization can be attributed to the presence of cobalt cupper phase". In the case of cupper cobalt phase, $\mathrm{Cu}$ ions exist in the +2 valence states. Also the magnetite phase (CuO) is considered as a ferrite compound [33]. In this case, there is a net magnetic moment because for the solid as a whole the spin moments are not completely cancelled; although the spin moments of the $\mathrm{Cu}^{2+}$ ions cancel one another, the magnetization in this phase arises from the parallel alignment of the moments of the $\mathrm{Cu}$ ions. The calculated values of $A$ and $\mathrm{K}$ are listed in table (3).

It can be noticed that sample (Mn-5, Fe-10) has the highest value of area, which is the indicator of the amount of heat produce, making it a very good candidate for hyperthermia application. Samples (Cu-15) and (Co-15) comes after it with values roughly half the value of (Co-5, Cu-10).

Table (3): Values of anisotropy energy density $(K)$ and area enclosed within the hysteresis loop (A) calculated from the magnetization curves.

\begin{tabular}{|c|c|c|}
\hline Sample & $\mathrm{K}(\mathrm{J} / \mathrm{Kg})$ & $\mathrm{A}(\mathrm{J} / \mathrm{Kg})$ \\
\hline $\mathrm{HA}$ & $0.25 \pm 0.02$ & $0.06 \pm 0.01$ \\
\hline $\mathrm{Cu}-15$ & $3.37 \pm 0.2$ & $0.21 \pm 0.02$ \\
\hline $\mathrm{Cu}-20$ & $1.57 \pm 0.1$ & $0.19 \pm 0.02$ \\
\hline $\mathrm{Co}-15$ & $2.28 \pm 0.2$ & $0.22 \pm 0.02$ \\
\hline Co-20 & $0.87 \pm 0.1$ & $0.11 \pm 0.01$ \\
\hline Co-5, Cu-10 & $7.79 \pm 0.3$ & $0.44 \pm 0.02$ \\
\hline
\end{tabular}

\section{Transmission electron microscopy (TEM) and Electron Diffraction pattern (EDP):}

The internal structure of the selected samples was studied via TEM technique. Figures (12), (13), (14) and (15) show the transmission electron micrographs and their corresponding electron diffractions of samples (HA), (Cu -15), (Co -15) and (Co-5, Cu-10) respectively. HA shows a homogenous structure with particle size ranging between 120 and 155 n.m. The EDP indicated the presence of bright dots on rings representing the planes of the hydroxyapatite crystal. This pattern is a typical pattern of polycrystalline bioceramics. In case of sample (Cu-15) the particle size ranges between 35 and 83 n.m, while in sample (Co-15) the particle size is in the range of $90 \mathrm{n} . \mathrm{m}$. It is noticed that in these two samples, the bright dots in 


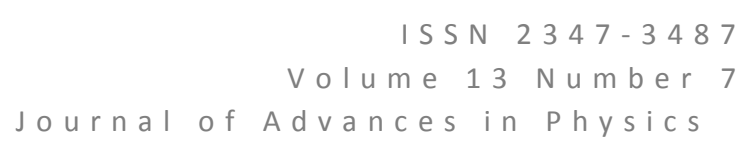

the diffraction pattern is arranged in more ordered lines. This may be explained in association with the XRD patterns of these samples which reveals the disappearance of apatite phase and the presence of the phase $(\mathrm{CuO})$ in case of $(\mathrm{Cu}-15)$ and cobalt oxide in case of (Co-15). The particle size of sample (Co-5, Cu-10) was between 55.4 and 73.4 n.m. The EDP of this sample showed a polycrystalline pattern due to the presence of many crystalline phases.
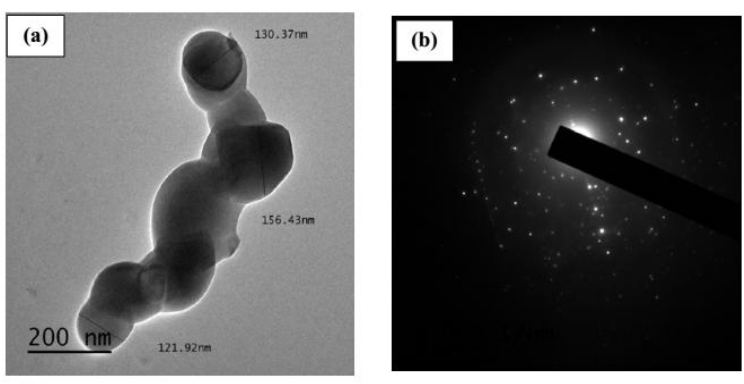

Figure 12:- TEM and EDP of pure hydroxyapatite
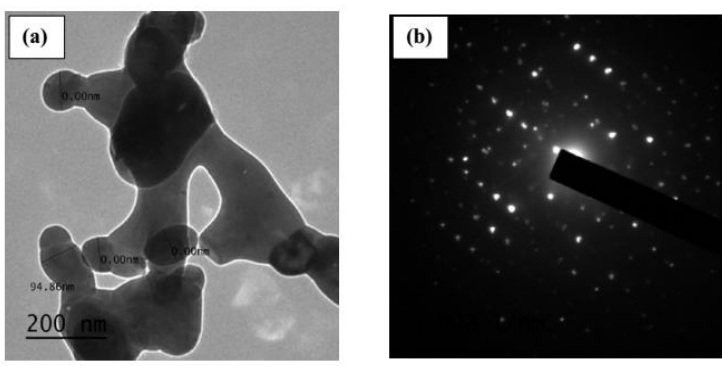
Figure 12:- TEM and EDP of Co-15 doped
hydroxyapatite
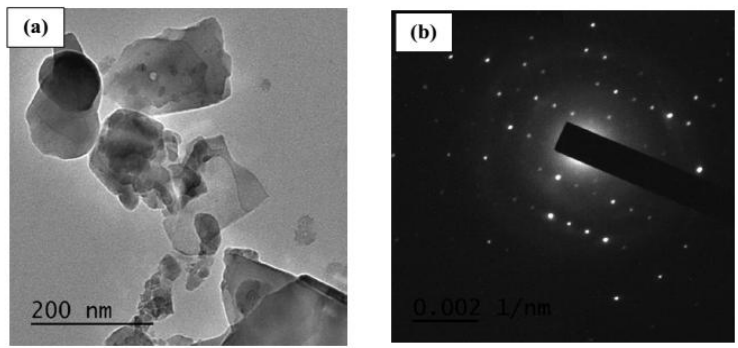

Figure 12:- TEM and EDP of Fe-15 doped hydroxyapatite
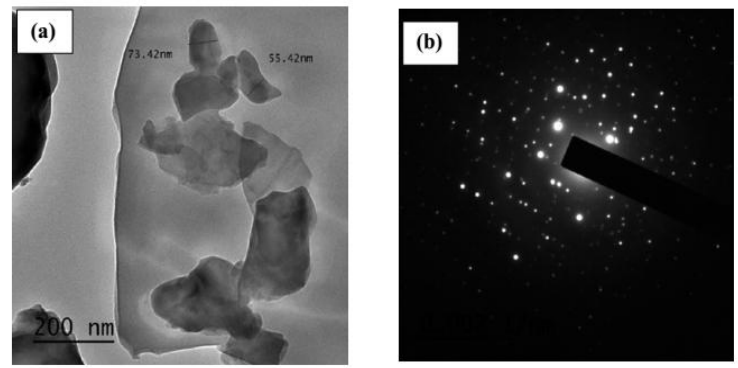

Figure 13:- TEM and EDP of Co-5, Cu-10 doped hydroxyapatite

\section{CONCLUSION}

The magnetic susceptibility of the prepared samples was found to increase linearly with the increase of the metal content in all samples indicating a paramagnetic behavior. Samples with the highest value of magnetic susceptibility were chosen for more study to test their ability for application in the field of hyperthermia treatment of bone tumors. Magnetization curves were obtained for these samples to study their behavior under the effect of magnetic field. The sample doped with copper and cobalt exhibited hysteresis loops which are characteristic for the magnetic materials. The samples were classified to be ferromagnetic material.

The sample (Co-5, Cu-10) had the highest values of saturation magnetization (MS), area $(A)$ enclosed within the hysteresis loop and magnetic anisotropy $(\mathrm{K})$. These parameters represent an indicator of the energy generated in the material under the effect of magnetic field, and hence the amount of heat produced by the sample. All these factors make sample (Co- 5, Cu- 10) a good candidate for hyperthermia application.

TEM and EDP techniques were used to study the internal structure of these samples. The micrographs and the diffraction patterns showed and confirmed the presence of crystal structures within the samples. The particle size was calculated from the micrographs and found to be in the range of nanometer for all the selected samples.

\section{REFERENCES}

1. Nandi, S., et al., Orthopaedic applications of bone graft \& graft substitutes: a review. 2010.

2. Dubok, V.A., Bioceramics-yesterday, today, tomorrow. Powder Metallurgy and Metal Ceramics, 2000. 39(7): p. 381-394.

3. Raucci, M.G., D. Giugliano, and L. Ambrosio, Fundamental Properties of Bioceramics and Biocomposites. Handbook of Bioceramics and Biocomposites, 2014: p. 1-19.

4. Gao, C., et al., Current progress in bioactive ceramic scaffolds for bone repair and regeneration. International journal of molecular sciences, 2014. 15(3): p. 4714-4732.

5. Bose, S., D. Banerjee, and A. Bandyopadhyay, Introduction to Biomaterials and Devices for Bone Disorders. Materials and Devices for Bone Disorders, 2016: p. 1.

6. Dorozhkin, S.V., Calcium orthophosphate bioceramics. Ceramics International, 2015. 41(10): p. 13913-13966. 


\section{ISSN $2347-3487$ \\ Volume $13 \mathrm{Number} 7$ \\ Journal of Advances in Physics}

7. D'Amora, U., et al., Hybrid Nanocomposites with Magnetic Activation for Advanced Bone Tissue Engineering. Bio-Inspired Regenerative Medicine: Materials, Processes, and Clinical Applications, 2016: p. 179.

8. Bañobre-López, M., et al., Hyperthermia induced in magnetic scaffolds for bone tissue engineering. IEEE Transactions on Magnetics, 2014. 50(11): p. 1-7.

9. Hurley, K.R., et al., Characterization of magnetic nanoparticles in biological matrices. 2015, ACS Publications.

10. Dutz, S. and R. Hergt, Magnetic particle hyperthermia-a promising tumour therapy? Nanotechnology, 2014. 25(45): p. 452001.

11. Ramakrishna, S., et al., Biomaterials: a nano approach. 2016: CRC Press.

12. Liu, Y., K. Ai, and L. Lu, Polydopamine and its derivative materials: synthesis and promising applications in energy, environmental, and biomedical fields. Chemical reviews, 2014. 114(9): p. 5057-5115.

13. Sadat-Shojai, M., et al., Synthesis methods for nanosized hydroxyapatite with diverse structures. Acta biomaterialia, 2013. 9(8): p. 7591-7621.

14. Tas, A.C., Use of Biomineralization Media in Biomimetic Synthesis of Hard Tissue Substitutes. Advances in Bioceramics and Biotechnologies II: Ceramic Transactions, Volume 247, 2014: p. 91-104.

15. Brown, W.E., et al., Octacalcium phosphate and hydroxyapatite: crystallographic and chemical relations between octacalcium phosphate and hydroxyapatite. Nature, 1962. 196(4859): p. 1050-1055.

16. LeGeros, R.Z., Calcium phosphate-based osteoinductive materials. Chemical reviews, 2008. 108(11): p. 47424753.

17. Zreiqat, $H_{\text {., }}$ et al., The incorporation of strontium and zinc into a calcium-silicon ceramic for bone tissue engineering. Biomaterials, 2010. 31(12): p. 3175-3184.

18. O'Donnell, M., et al., Structural analysis of a series of strontium-substituted apatites. Acta Biomaterialia, 2008. 4(5): p. 1455-1464.

19. Bose, S. and S. Tarafder, Calcium phosphate ceramic systems in growth factor and drug delivery for bone tissue engineering: a review. Acta biomaterialia, 2012. 8(4): p. 1401-1421.

20. Boanini, E., M. Gazzano, and A. Bigi, lonic substitutions in calcium phosphates synthesized at low temperature. Acta biomaterialia, 2010. 6(6): p. 1882-1894.

21. Theron, J., J. Walker, and T. Cloete, Nanotechnology and water treatment: applications and emerging opportunities. Critical reviews in microbiology, 2008. 34(1): p. 43-69.

22. DeNardo, S.J., et al., Development of tumor targeting bioprobes (111/n-chimeric L6 monoclonal antibody nanoparticles) for alternating magnetic field cancer therapy. Clinical Cancer Research, 2005. 11(19): p. 7087s7092s.

23. Shiba, H., Magnetic susceptibility at zero temperature for the one-dimensional Hubbard model. Physical Review B, 1972. 6(3): p. 930.

24. Stouff, P. and J. Boulègue, Geochemistry and crystallochemistry of oceanic hydrothermal manganese oxyhydroxides showing Mn-Cu association. Geochimica et Cosmochimica Acta, 1989. 53(4): p. 833-843.

25. Sutter, B., et al., Characterization of iron, manganese, and copper synthetic hydroxyapatites by electron paramagnetic resonance spectroscopy. Soil Science Society of America Journal, 2002. 66(4): p. 1359-1366.

26. Kostakis, G.E., et al., High-nuclearity cobalt coordination clusters: Synthetic, topological and magnetic aspects. Coordination Chemistry Reviews, 2012. 256(11): p. 1246-1278.

27. Schenck, J.F., The role of magnetic susceptibility in magnetic resonance imaging: MRI magnetic compatibility of the first and second kinds. Medical physics, 1996. 23(6): p. 815-850.

28. Sharifi, I., H. Shokrollahi, and S. Amiri, Ferrite-based magnetic nanofluids used in hyperthermia applications. Journal of Magnetism and Magnetic Materials, 2012. 324(6): p. 903-915.

29. Cullity, B.D. and C.D. Graham, Introduction to magnetic materials. 2011: John Wiley \& Sons.

30. Goya, G., et al., Static and dynamic magnetic properties of spherical magnetite nanoparticles. Journal of Applied Physics, 2003. 94(5): p. 3520-3528.

31. Gould, C., et al., Tunneling anisotropic magnetoresistance: a spin-valve-like tunnel magnetoresistance using a single magnetic layer. Physical review letters, 2004. 93(11): p. 117203.

32. Chiba, D., et al., Magnetization vector manipulation by electric fields. Nature, 2008. 455(7212): p. 515.

33. Jacob, K., K. Fitzner, and C. Alcock, Activities in the spinel solid solution, phase equilibria and thermodynamic properties of ternary phases in the system Cu-Fe-0. Metallurgical and Materials Transactions B, 1977. 8(2): p. $451-460$. 